\title{
Association between increased magnetic susceptibility of deep gray matter nuclei and decreased motor function in healthy adults
}

\author{
Wei Li ${ }^{\text {a,e,f }}$, Christian Langkammer ${ }^{c}$, Ying-Hui Chou ${ }^{a}$, Katja Petrovic ${ }^{c}$, Reinhold Schmidt ${ }^{c}$, Allen W. Song a,b, \\ David J. Madden ${ }^{\mathrm{a}, \mathrm{d}}$, Stefan Ropele ${ }^{\mathrm{c}, *}$, Chunlei Liu ${ }^{\mathrm{a}, \mathrm{b}, * *}$
}

\footnotetext{
a Brain Imaging and Analysis Center, Duke University Medical Center, Durham, NC 27710, USA

b Department of Radiology, Duke University Medical Center, Durham, NC 27710, USA

c Department of Neurology, Medical University of Graz, Graz, Austria

d Department of Psychiatry and Behavioral Sciences, Duke University Medical Center, Durham, NC 27710, USA

e Research Imaging Institute, University of Texas Health Science Center at San Antonio, TX 78229, USA

${ }^{\mathrm{f}}$ Department of Ophthalmology, University of Texas Health Science Center at San Antonio, TX 78229, USA
}

\section{A R T I C L E I N F O}

\section{Article history:}

Accepted 5 October 2014

Available online 12 October 2014

\section{Keywords:}

Magnetic susceptibility

Brain iron

Aging

Motor control

Cognitive function

\begin{abstract}
A B S T R A C T
In the human brain, iron is more prevalent in gray matter than in white matter, and deep gray matter structures, particularly the globus pallidus, putamen, caudate nucleus, substantia nigra, red nucleus, and dentate nucleus, exhibit especially high iron content. Abnormally elevated iron levels have been found in various neurodegenerative diseases. Additionally, iron overload and related neurodegeneration may also occur during aging, but the functional consequences are not clear. In this study, we explored the correlation between magnetic susceptibility - a surrogate marker of brain iron - of these gray matter structures with behavioral measures of motor and cognitive abilities, in 132 healthy adults aged $40-83$ years. Latent variables corresponding to manual dexterity and executive functions were obtained using factor analysis. The factor scores for manual dexterity declined significantly with increasing age. Independent of gender, age, and global cognitive function, increasing magnetic susceptibility in the globus pallidus and red nuclei was associated with decreasing manual dexterity. This finding suggests the potential value of magnetic susceptibility, a non-invasive quantitative imaging marker of iron, for the study of iron-related brain function changes.
\end{abstract}

(c) 2014 Elsevier Inc. All rights reserved.

\section{Introduction}

Iron is essential for normal functioning of the brain, because it is a co-factor for many enzymes involved in oxygen transport, neurotransmitter synthesis, electron transfer, and myelin production (Moos and Morgan, 2004; Zecca et al., 2004). Plasma iron is not directly available for neuronal cells, but is moved across the blood brain barrier to the neuronal cells through specific transport mechanisms (Burdo and Connor, 2003). Brain tissues store the iron in the forms of ferritin or hemosiderin. Iron and iron storage proteins such as ferritin are found not only in oligodendrocytes but also in microglial cells in the brain. The rates of iron uptake and storage vary significantly across brain regions (Hallgren and Sourander, 1958; Haacke et al., 2005). Specifically, a number of deep brain gray matter structures, including putamen (PU), globus pallidus (GP), caudate nuclei (CN), red nuclei (RN), substantia

\footnotetext{
* Correspondence to: S. Ropele, Department of Neurology, Medical University of Graz, Auenbruggerplatz 22, $8036 \mathrm{Graz}$, Austria.

** Correspondence to: C. Liu, Brain Imaging and Analysis Center, Duke University School of Medicine, 2424 Erwin Road, Suite 501, Durham, NC 27705, USA. C. Liu).
}

nigra (SN), dentate nuclei (DN), and others, have much higher iron contents than surrounding tissues (Hallgren and Sourander, 1958). Despite the many important physiological functions of iron, excessive iron can stimulate oxidative damage through the generation of toxic free radicals (Emerit et al., 2001). There is increasing evidence that excessive iron accumulation, especially in the iron rich gray matter regions, is closely involved in mechanisms associated with neurodegeneration in aging, such as Parkinson's disease and Alzheimer's disease (Zecca et al., 2004).

Deposition of paramagnetic non-heme iron in brain tissue can lead to a reduction of $12 *$ relaxation rate and altered phase contrast that is measurable by gradient echo (GRE) MRI. While the reduction in T2* has long been used as a measure of brain iron (Gelman et al., 1999; Aquino et al., 2009), the recently developed technique of quantitative susceptibility mapping (QSM) allows the quantification of tissue magnetic susceptibility from GRE signal phase (Liu et al., 2009; Shmueli et al., 2009; de Rochefort et al., 2010; Wharton et al., 2010; Li et al., 2011; Schweser et al., 2011; Wu et al., 2012). The resultant tissue magnetic susceptibility provides a novel contrast with high sensitivity to brain iron. Similar to R2* (1/T2*), accumulating evidence suggests that the magnetic susceptibility of gray matter is linearly proportional to the iron content (Shmueli et al., 2009; Schweser et al., 2011; Bilgic et al., 2012; Langkammer et al., 2012; Wu et al., 2012). Magnetic 
susceptibility is a basic physical property of matter, independent of magnetic field strength, whereas R2* scales linearly with field strength (Yao et al., 2009). In addition, magnetic susceptibility mapping typically provides a higher contrast-to-noise ratio and clearer gray-white matter tissue boundary (Rauscher et al., 2005; Li et al., 2011; Deistung et al., 2013; Li et al., 2014b). Thus, QSM is a promising candidate for noninvasive assessment of brain iron.

Early histochemical work revealed that brain iron increases with age (Hallgren and Sourander, 1958). A recent study evaluated the evolution of magnetic susceptibility as a function of age in a cohort of 191 healthy participants from 1 to 83 years of age (Li et al., 2014b). The results indicated that susceptibility of iron-rich, deep gray matter follows an exponential growth trajectory, which agrees well with the known characteristics of brain iron accumulation. Interestingly, MR susceptibilities of these nuclei also exhibited a wider variation of iron content at older ages (e.g., $>40$ years of age). While existing studies have so far demonstrated the anatomical variations and age-related evolution of magnetic susceptibility, it is unclear if these variations in susceptibility have any functional significance.

Given the role of iron overload in tissue degeneration and the association of deep gray matter in motor function, we hypothesized that in healthy adults, increased magnetic susceptibility of the iron-rich, deep gray matter nuclei is correlated with decline of motor functions, independently of age. In this study, we examined the relation between susceptibility and behavioral performance measures in a cohort of 132 healthy, community-dwelling adults between 40 and 83 years of age. The mean magnetic susceptibility of six subcortical gray matter regions was measured. Participants performed a series of behavioral tests targeting various cognitive and motor abilities. We performed factor analysis to identify the latent variables of the test scores, and correlated the factor scores with the magnetic susceptibility of the six deep gray matter regions.

\section{Materials and methods}

\section{Participants}

The study cohort was from the Austrian Stroke Prevention Family Study (ASPS-Fam), a prospective, single-center, community-based study on the cerebral effects of vascular risk factors in healthy older adults of the city of Graz, Austria. The ASPS-Fam represents an extension of the Austrian Stroke Prevention Study (ASPS) which was established in 1991 (Schmidt et al., 1999; Schmidt et al., 2003; Schmidt et al., 2005). Between 2006 and 2013, study participants of the ASPS and their first grade relative were invited to enter ASPS-Fam. Inclusion criteria were no history of previous stroke or dementia and a normal neurologic examination. A total of 381 individuals were included. The entire cohort underwent an extended diagnostic work-up including clinical history, blood tests (including blood iron levels), cognitive testing and a thorough vascular risk factor assessment. All individuals underwent MRI, except for 26 individuals who had contraindications. Magnetic susceptibility imaging was available in a total of 135 participants $40-83$ years of age at the time of this study. Three participants were excluded due to the following reasons: (1) missing data from several behavioral tests; (2) obvious signs of bleeding in GP; and (3) poor image quality. Therefore, the total number of participants reduced to $132(M=64.50, S D=10.64)$, with mean Mini-Mental State Exam (MMSE) score (Folstein et al., 1975) of 28.19 $(S D=1.46)$. Women were $59 \%(n=78)$ of the sample. The study protocol was approved and accepted by the ethics committee of the Medical University of Graz, Austria, and an informed consent was obtained from all participants.

\section{Brain imaging}

Each participant was scanned on a 3T scanner with a 12-channel receive array coil at the Medical University of Graz. A spoiled 3D multi- echo gradient-echo sequence (FLASH) was used for quantitative susceptibility mapping. The scan parameters were: in-plane resolution $=$ $0.9 \times 0.9 \mathrm{~mm}^{2}$, matrix $=256 \times 208$, flip angle $=20^{\circ}$, TE of first echo $=4.92 \mathrm{~ms}$, echo spacing $=4.92 \mathrm{~ms}$, and number of echoes $=6$. The slice thickness was either $4 \mathrm{~mm}$ with $\mathrm{TR}=68 \mathrm{~ms}$ or $2 \mathrm{~mm}$ with $\mathrm{TR}=35 \mathrm{~ms}$, respectively. The entire brain was covered by the FLASH sequence. Previous studies confirmed that 2-mm and 4-mm slice thicknesses do not produce significantly different magnetic susceptibility values of the iron rich deep gray matter structures (Li et al., 2013b).

To determine the nonlinear warping matrix between the individual participant space and the MNI space, T1-weighted images of the same participants were acquired using a 3D MPRAGE sequence. The images were acquired in the sagittal view with the following parameters: data matrix $=224 \times 256 \times 176,1 \mathrm{~mm}$ isotropic resolution, flip angle $=9^{\circ}$, $\mathrm{TI}=900 \mathrm{~ms}, \mathrm{TE}=2.19 \mathrm{~ms}$ and $\mathrm{TR}=1900 \mathrm{~ms}$.

\section{Quantitative susceptibility mapping}

We performed quantitative susceptibility mapping as described previously (Li et al., 2011) under the software STI Suite (Duke University) (Li et al., 2014a). Briefly, the brain was extracted from the magnitude using the brain extraction tool in FSL (Smith, 2002). Phase maps were unwrapped using a Laplacian-based phase unwrapping method ( $\mathrm{Li}$ et al., 2011). The unwrapped phase maps from all echoes were then normalized by the corresponding echo times to yield the frequency shift using the following equation:

$\Delta f=\sum_{e=1}^{n e} \varphi_{e} / \sum_{e=1}^{n e} T E_{e}$

Here, $n e$ is the number of echoes; $\varphi$ is the image phase and $\Delta f$ is the frequency shift. This equation assumes linear evolution of phase contrast and assigns different weights for different echoes according to their TEs. This approach is valid for the evaluation of magnetic susceptibility of gray matter structures, with the advantage of higher signal-tonoise level than susceptibility mapping using only a single echo. The background frequency was removed using a variable-filter-radius SHARP method (Schweser et al., 2011). Specifically, the diameter of the spherical mean filter decreases from a maximum value of $25 \mathrm{~mm}$ towards $1 \mathrm{~mm}$ at the brain boundary (Li et al., 2011; Wu et al., 2012). Susceptibility maps were then derived from the brain tissue frequency shift using the LSQR method (Li et al., 2011).

\section{Regions of interest}

We obtained the deep gray matter regions of interest (ROIs) by warping a customized common atlas created in the MNI space to each individual participant space using FSL (FMRIB, University of Oxford, UK). A schematic diagram describing this procedure is shown in Fig. 1. Briefly, we registered the T1-weighted images to the standard template (MNI152_T1_1mm) using FNIRT. We then used the resulting registration matrices to wrap the susceptibility maps to the MNI space and then averaged the maps to generate mean susceptibility. We initially created the atlas for the deep gray matter nuclei using the Harvard-Oxford subcortical atlas and Talairach atlas in FSL; we then manually refined them based on the structural boundaries shown in the mean susceptibility maps.

Examples of the six ROIs, and their associated mean magnetic susceptibility values, are presented in Fig. 2. Six major deep gray matter nuclei were segmented, specifically the PU, GP, CN, RN, SN, and DN (Fig. 2, Panels A-C). This study-specific common atlas for deep gray matter nuclei was then warped to each individual participant space using FSL. The ROIs of each participant were visually examined and minor refinement of the ROIs was made when necessary. As illustrated in Fig. 2, Panels D and $\mathrm{E}$, the ROIs varied in size as well as the mean level of magnetic 
A

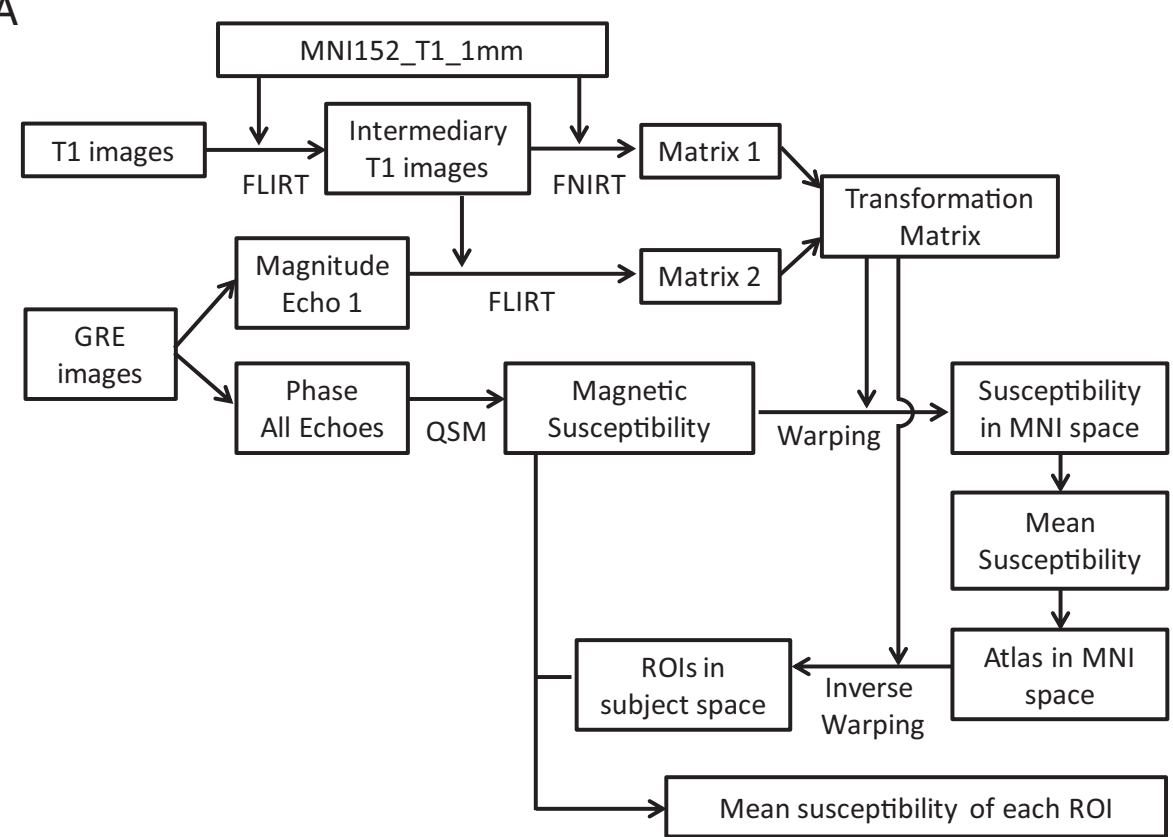

B
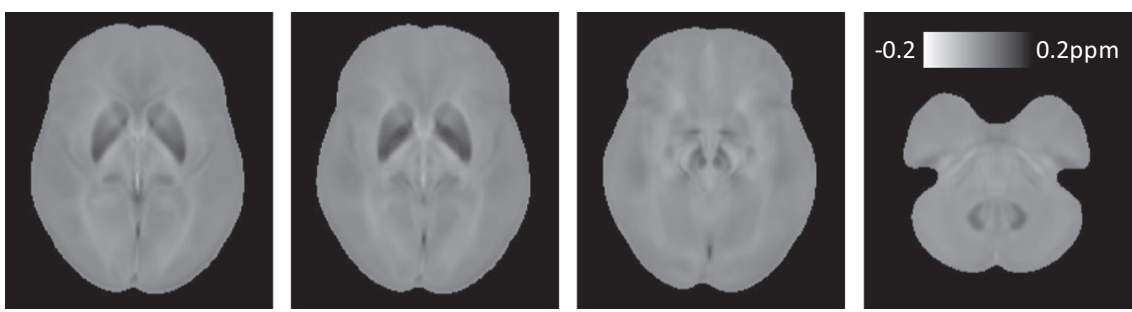

Fig. 1. A schematic representation of the image analysis pipeline (A) and the mean magnetic susceptibility maps in different axial locations (B).

susceptibility. Thus, as noted in the Results section, the susceptibility values were adjusted statistically for ROI size.

\section{Behavioral tests}

Each participant completed a series of behavioral tests: perceptual motor functioning, verbal working memory, executive functioning, and verbal fluency within one month of MRI scan. These tests yielded a total of 11 dependent variables, as described as follows.

Purdue-pegboard-test (Lafayette instrument company, USA)

This test is a clinical measure used to assess manual dexterity and perceptual-motor speed (Tiffin and Asher, 1948). The pegboard features two parallel lines of holes running vertically down the center of
A

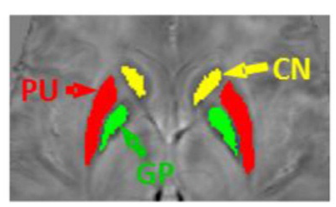

D



B

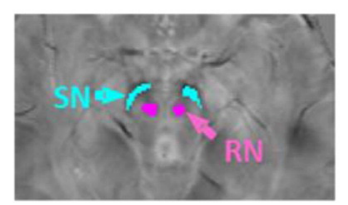

C

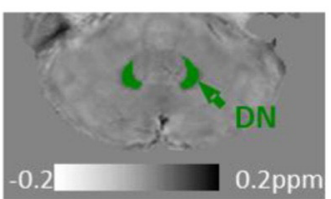

$E$

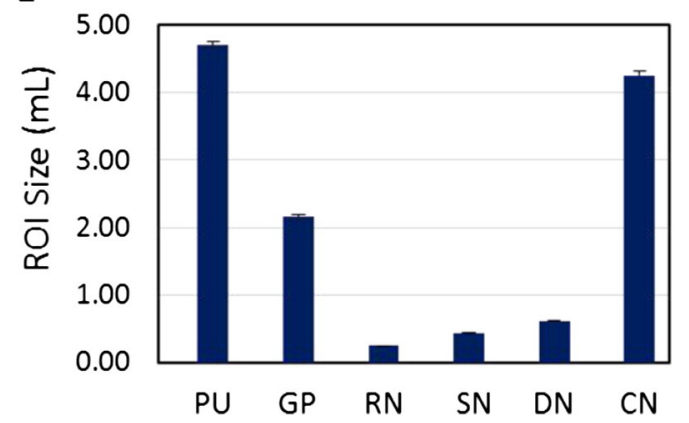

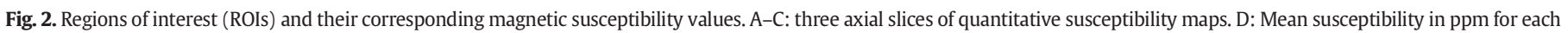
ROI, + 1 SE; E: Mean size in $\mathrm{mL}$ for each ROI, +1 SE. PU = putamen; GP = globus pallidus; $\mathrm{RN}=$ red nuclei; $\mathrm{SN}=$ substantia nigra; $\mathrm{DN}=$ dentate nuclei; $\mathrm{CN}=$ caudate nuclei. 
the board. This test consisted of four subtests. In the first portion of the test, a participant was given $30 \mathrm{~s}$ to place as many pins in the holes as possible using the right hand. In the second portion of the test, this process was repeated using the left hand. In the third portion of the test, the participant had another $30 \mathrm{~s}$ to place pins into the holes using both hands simultaneously. In the final portion of the test, usually called the assembly test, the participant must assemble pins, washer, and collar in a fixed order using the left and the right hand alternatively. The participant had $60 \mathrm{~s}$ to complete as many assembles as possible. Thus, the Purdue pegboard test yielded four dependent variables: a) pins/30 s right; b) pins/30 s left; c) pins/30 s both; d) assembles/60 s assembly.

\section{Digit span test}

This subtest of the Wechsler Adult Intelligence Scale IV is composed of digits-forward and digits-backward tasks, and is used to measure verbal working memory (Wechsler, 2003). In these tasks, participants listened to a series of discrete digits and subsequently recalled the series of digits in the same (i.e., digits-forward task) or reverse (i.e., backward digit span task) order of presentation. Each task comprised eight difficulty levels with two trials for each level. The test started with a two-digit sequence, and the series of digits were subsequently increased by one digit every two trials. The task was discontinued if the participant failed to recall two consecutive series with the same number of digits. Each correct trial is awarded a score of 1 , with a total maximum score of 16 points. The digit span test yielded two measures of memory span: digits-forward score and digitsbackward score.

\section{Wisconsin Card Sorting Test (WCST, Heaton et al., 1993)}

This test was designed to measure the ability to display flexibility in the face of changing rules. It is assumed that successful completion of this test requires engagement of executive functions (Jodzio and Biechowska, 2010). During this task, four stimulus cards with shapes on them were presented to the participant. The stimulus cards differed in color, number, and the form of the shapes. The participant was asked to match a series of cards to one of the four stimulus cards based on one of the rules (color, number or form of shape). The correct rule was not revealed to the participant. Instead, the participant was instructed to determine the rule based on the feedback (right or wrong) he/she received upon response and was told that the rule might change without notice. The rule actually changed every time the participant completed ten consecutive correct trials. The WCST yielded three dependent variables: 1 ) the number of categories completed, 2) the number of perseverative errors (i.e., errors due to a failure to switch from the previous rule), and 3 ) the sum of non-perseverative and perseverative errors.

Trail making test B (War Department Adjutant General's Office, 1944; Reitan and Wolfson, 1985)

This test measures executive functioning, psychomotor speed, and visual scanning. Participants were instructed to draw lines to connect 25 circled numbers and letters in an alternating numeric and alphabetic sequence (i.e., 1-A-2-B) as rapidly as possible. The dependent variable was the time in seconds to finish the test.

\section{Semantic fluency test}

To measure semantic fluency, we tabulated the number of supermarket items the participants named in 1 min ("name things you can buy in a large supermarket in $1 \mathrm{~min}$ ") (Lezak, 1995). The dependent variable was the number of items named over $1 \mathrm{~min}$.

Mean values of the behavioral tests, and clinical measures of blood iron levels, are presented in Table 1.
Table 1

Behavioral and clinical test scores.

\begin{tabular}{lrr}
\hline Variable & \multicolumn{1}{c}{$M$} & \multicolumn{1}{c}{$S D$} \\
\hline Purdue pegboard: right (number completed) & 12.58 & 2.46 \\
Purdue pegboard: left (number completed) & 12.30 & 2.27 \\
Purdue pegboard: left + right (number completed) & 10.29 & 2.21 \\
Purdue pegboard: assembly (number completed) & 24.89 & 7.31 \\
Digit span: forward (items) & 6.20 & 1.14 \\
Digit span: backward (items) & 4.25 & 1.32 \\
WCST: errors & 18.39 & 12.25 \\
WCST: perseverative responses & 7.04 & 4.53 \\
WCST: categories & 5.66 & 1.00 \\
Trails B (sec) & 115.10 & 58.90 \\
Word fluency (items/min) & 23.99 & 6.93 \\
Iron $(\mu \mathrm{d} / \mathrm{dl})$ & 105.48 & 32.04 \\
Transferrin $(\mathrm{mg} / \mathrm{dl})$ & 252.74 & 44.85 \\
Ferritin $(\mu \mathrm{g} / \mathrm{L})$ & 130.41 & 109.96 \\
\hline
\end{tabular}

Note: $n=132$. WCST $=$ Wisconsin Card Sorting Test.

\section{Results}

\section{Magnetic susceptibility}

Because the ROIs varied widely in size (i.e. the volumes of the deep brain nuclei) (Fig. 2), all analyses of the magnetic susceptibility values were adjusted for ROI size in the following manner. For each ROI, we created a regression model in which ROI size was a predictor of susceptibility. We then used the residual of this model, for each participant, for use in further analyses. Because the residuals are by definition uncorrelated with size, this method created a size-independent measure of susceptibility for each ROI. We then formed standardized ( $z$ ) scores of these size-residualized susceptibility values for each ROI, so that the ROI susceptibility values would have a common scale. Further, preliminary analyses indicated no reliable effects of gender, but gender was added as a covariate to all analyses.

We have previously shown that the susceptibility of several deep gray matter nuclei evolves exponentially as a function of age (Li et al., 2013b). Susceptibility typically increases nonlinearly at young ages (1-20 years), then either approaching a plateau or increasing linearly at older ages ( $>40$ years). Across these six ROIs, an age-related increase in magnetic susceptibility was evident within the putamen (Fig. 3, Panel A), but the effect of age was not significant within any of the other ROIs. Further analyses of the susceptibility values included age as a covariate.

\section{Relation between behavioral performance and magnetic susceptibility}

Prior to analysis, all demographic and behavioral variables were transformed to $\mathrm{z}$ scores based on the sample mean and standard deviation per variable. We performed an exploratory factor analysis of the 11 behavioral measures to identify the independent, latent variables of behavioral performance. We then used the factor scores representing these latent variables as dependent variables in regression analyses with participants' years of age and size-residualized susceptibility from the six ROIs as predictors.

Results of the factor analysis revealed that the eigenvalues of the first two factors were greater than 1.0, and these factors accounted for $84 \%$ of the common variance. Following Varimax rotation, we used 0.60 as a threshold for significant factor loadings (Table 2), on the basis of sample size and number of variables (Buja and Eyuboglu, 1992). The first factor comprised all four variables from the Purdue pegboard test, and we interpret this factor as representing manual dexterity. The second factor comprised two variables from the Wisconsin Card Sorting Test, and we interpret this factor as representing executive function. We conducted correlations between the factor scores and age, partialed for the effects of gender and global cognitive functioning (MMSE). The first factor representing manual dexterity exhibited significant decline as a function of increasing age (Fig. 4, Panel A), whereas the second factor 
A

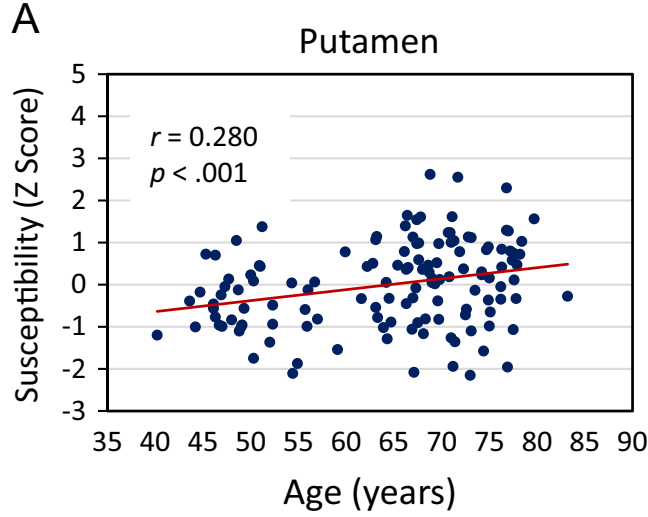

C

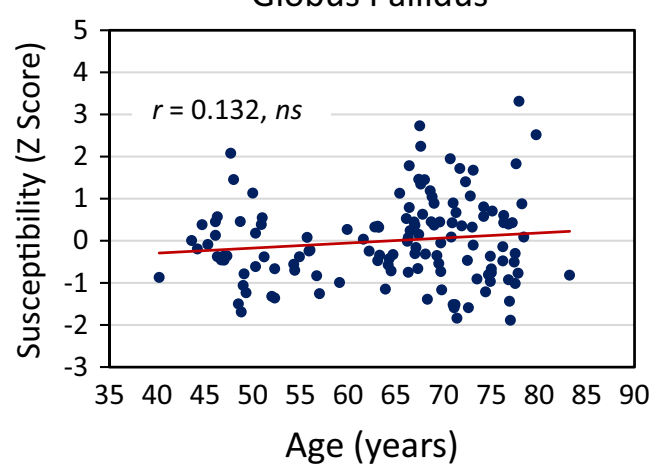

$E$

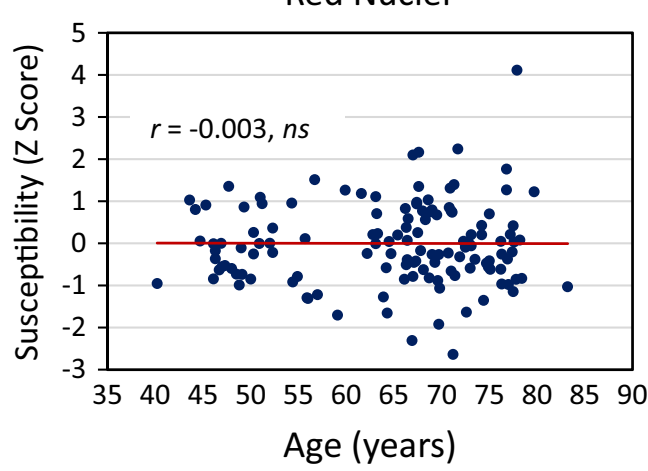

B

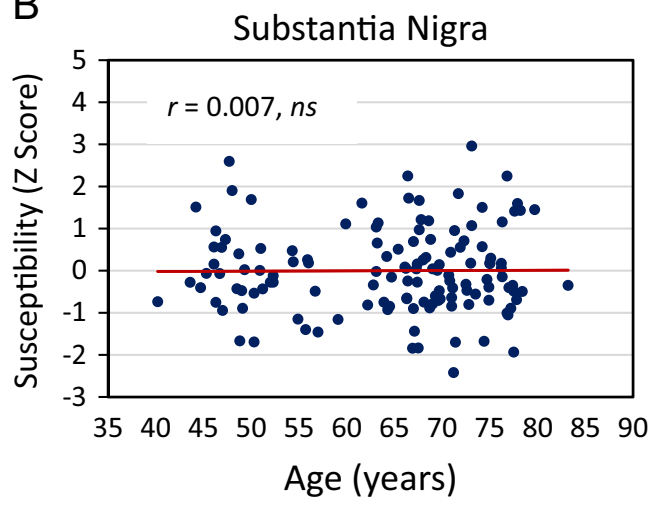

Dentate Nuclei



$\mathrm{F}$

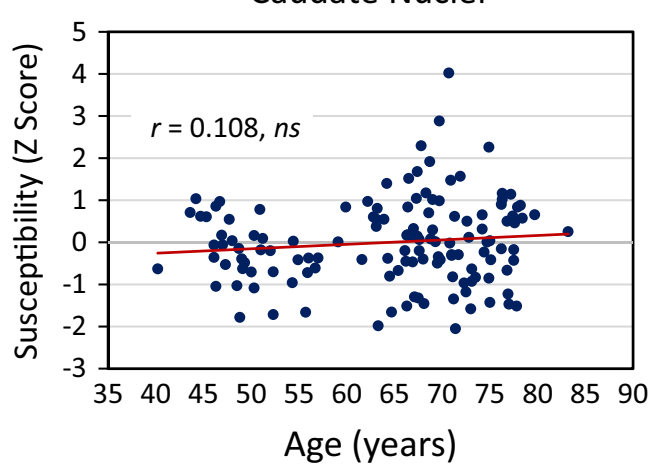

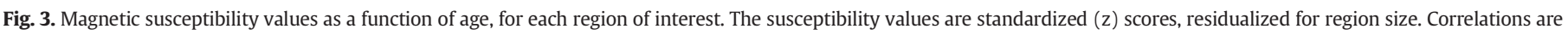
partialed for gender. A significant age-related increase in susceptibility was evident only within the putamen.

Table 2

Rotated factor loadings for factors 1 and 2 from an exploratory factor analysis of 11 behavioral tests.

\begin{tabular}{lcc}
\hline Behavioral Tests & $\begin{array}{l}\text { Factor } 1 \\
\text { (Manual dexterity) }\end{array}$ & $\begin{array}{l}\text { Factor } 2 \\
\text { (Executive function) }\end{array}$ \\
\hline Purdue right & $\mathbf{0 . 7 6 8}$ & 0.112 \\
Purdue left & $\mathbf{0 . 8 6 4}$ & -0.002 \\
Purdue both & $\mathbf{0 . 8 1 9}$ & 0.018 \\
Purdue assembly & $\mathbf{0 . 7 6 6}$ & 0.068 \\
Digit symbol forward & 0.187 & 0.084 \\
Digit symbol backward & 0.092 & 0.112 \\
WCST error & -0.083 & $-\mathbf{0 . 6 8 2}$ \\
WCST category & 0.052 & $\mathbf{0 . 8 5 4}$ \\
WCST perseverative error & 0.019 & -0.291 \\
Trail making & -0.422 & -0.221 \\
Semantic fluency & 0.340 & 0.007 \\
\hline
\end{tabular}

Note: $n=132$. Factor loadings $>0.60$ are presented in bold. WCST $=$ Wisconsin Card Sorting Test. representing executive function did not exhibit significant age-related decline (Fig. 4, Panel B).

The bivariate correlations between susceptibility and the behavioral factor scores, for each ROI considered independently, are presented in Table 3. These correlations are partialed for the effects of age, gender, and global cognitive function (MMSE). For manual dexterity, a decreasing behavioral score was associated with increasing magnetic susceptibility in both the GP and RN (Fig. 5). Given the relation between magnetic susceptibility and manual dexterity for these two ROIs, we explored potential age-related differences in this pattern. We split the sample into two groups at the median age value (67.4 years) and performed bivariate susceptibility-dexterity correlations within each sub-group, partialed for age, gender, and MMSE. For the younger group $(n=67)$, the susceptibility-dexterity correlation was significant for the GP, $r=$ $-0.318, p<0.01$, but not for the RN, $r=-0.137, p=0.28$, whereas for the older group ( $n=65$ ), the correlation was significant for the RN, 
A

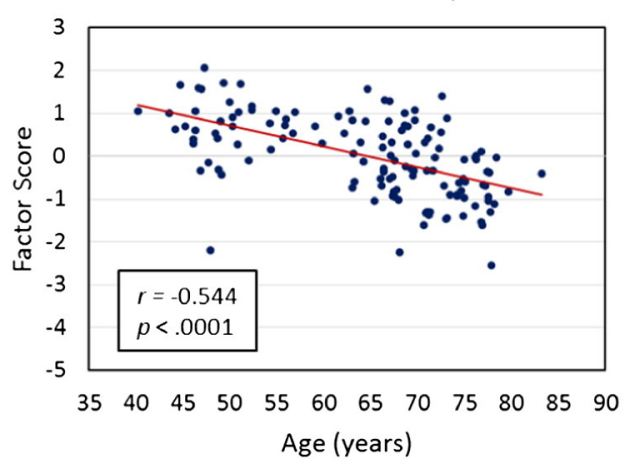

B



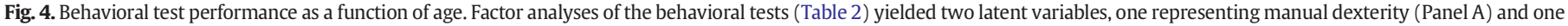


only for Factor 1 (manual dexterity).

$r=-0.262, p<0.05$, but not for the GP, $r=-0.205, p=0.11$. For each ROI, however, direct comparison of the correlations for the two subgroups (with Fisher's $r$ to $z$ transformation), was not significant, indicating that the variation in the correlations across the age sub-groups was not reliable.

We further obtained an estimate of age-related differences in region size by standardizing each ROI size in $\mathrm{mL}$ to the sample mean and standard deviation and then averaging these standard ( $\mathrm{z}$ ) scores across participants. The average ROI size decreased as a function of increasing age, $r=-0.198, p<0.05$, but average ROI size was unrelated to either MMSE score, the manual dexterity factor, or the executive function factor, $p>0.20$ in each case. For those ROIs exhibiting a correlation with behavior (GP and RN), no significant correlations were observed with either age or the behavioral measures.

The magnetic susceptibility values were all correlated positively across the ROIs, with bivariate correlations ranging from $r=+0.197$, $p<0.05$, to $r=+0.571, p<0.001$, across the 15 pairs of ROIs. We thus conducted two linear regression analyses, one for each of the latent variables from the factor analysis, representing manual dexterity and executive function, with all of the ROI values entered simultaneously, thus controlling the shared variance among the ROIs. Each regression model contained nine predictor variables: the six susceptibility values from the six ROIs, plus three demographic variables (gender, age, and MMSE) to control for individual differences theoretically unrelated to susceptibility and the behavioral outcome measure.

In the regression analysis of the first behavioral factor (manual dexterity), the nine predictors combined accounted for $37 \%$ of the variance in manual dexterity, $F(9,122)=7.82, p<0.0001$ (Table 4). Age was a significant predictor, reflecting the age-related decline in this measure. Among the six susceptibility variables, only the GP was a significant predictor, independent of the other ROIs. Increasing magnetic susceptibility within the GP was associated with decreasing manual dexterity (Fig. 5, Panel A). In the regression analysis of the second behavioral factor (executive function), the overall model with all predictors was not

Table 3

Correlations of magnetic susceptibility with manual dexterity and executive function.

\begin{tabular}{lll}
\hline Region & Manual dexterity & Executive function \\
\hline Putamen & -0.058 & -0.047 \\
Globus pallidus & $-0.216^{* *}$ & -0.077 \\
Red nuclei & $-0.199^{*}$ & -0.120 \\
Substantia nigra & -0.074 & -0.099 \\
Dentate nuclei & -0.062 & -0.158 \\
Caudate nuclei & 0.028 & 0.036 \\
\hline
\end{tabular}

Note. $n=132$. Values are Pearson $r$ correlations, partialed for the effects of age, gender, and global cognitive function (MMSE). Magnetic susceptibility values were residualized for the effects region size.

$* p<0.05$.

$* * \quad p<0.01$. significant, $F(9,122)<1.0$, and none of the individual predictors was significant (Table 5).

Correlations conducted between the three biomarkers of peripheral blood iron and the susceptibility values did not yield any significant effects (Table 6).

\section{Discussion}

In this study, we investigated the relationship between magnetic susceptibility of iron rich deep gray matter and brain functions measured by behavioral tests. Factor analysis of the behavioral tests identified two latent variables: manual dexterity and executive function. The manual dexterity factor was more sensitive than executive function to the effects of magnetic susceptibility. We found that, controlling for individual differences in gender, age, and global cognitive function, decreased manual dexterity was significantly correlated with increased magnetic susceptibility of GP and RN, but not the susceptibility of other gray matter structures (Table 3 and Fig. 5). When controlling for the shared variance among the ROIs, the age-related effect remained significant for the GP, but not for the RN (Tables 4 and 5), indicating a stronger effect for the GP. As the GP is the gray matter structure containing the highest amount of non-heme iron (Fig. 2, Panel D), these results suggest that high iron contents in GP can predispose individuals to higher risk of iron-overload induced motor function decline.

While the six different gray matter nuclei share a common feature of high iron content, they belong to different structural and functional units of the brain. The CN, PU, GP and SN are the main components of the basal ganglia, which are known to play major roles in regulating and modulating motor control functions through the widespread interconnections with the neocortex and the prefrontal and premotor cortices (Kandel and Schwartz, 1985). Consistent with this knowledge, we observed significant correlations between the manual dexterity factor score and the magnetic susceptibility of GP. These results suggest that iron overload in GP is associated with a negative impact on brain motor functions. Overall, our results are in good agreement with a previous exploratory study using 10 participants indicating that the fielddependent R2 increase (FDRI) values, another measure of brain iron, of basal ganglia nuclei also correlated with motor performance (Sullivan et al., 2009). It is worth noting that our results did not reveal a significant correlation between the magnetic susceptibility of SN and motor function scores, although it is well known that the motor function decline in Parkinson's disease is associated with dopamine cell loss and increased iron content in SN. A previous study also documented the increased magnetic susceptibility in Parkinson's disease (Lotfipour et al., 2012). It is possible that the iron accumulation in SN during normal aging in our study group did not reach the extent as found in pathological tissue damage. 
A



$\mathrm{B}$

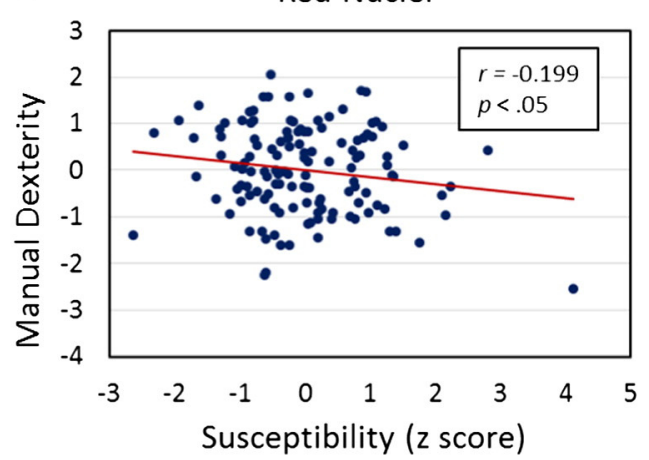


residualized for region size. Correlations were partialed for age, gender, and global cognitive function (MMSE).

The RN is a prominent brain stem structure, which is dorsally located to SN but is involved in different neuronal networks from that of basal ganglia. The origin of RN is suggested to be related to the advent of limbs, or limb-like structures during evolution. The RN plays important roles in motor control functions in lower vertebrates, but its importance in modulating the motor functions in human is less confirmed (ten Donkelaar, 1988; Gruber and Gould, 2010). It has been suggested to be the backup to the corticospinal tract through the connections with the dentate nucleus, the motor cortices and the spinal cord (Nioche et al., 2009; Gruber and Gould, 2010). There also have been studies suggesting that the RN increases its function to fulfill cerebellar compensation in the presence of basal ganglia dysfunction (Lewis et al., 2013). Although increased magnetic susceptibility in the RN did exhibit a significant relation to decreased manual dexterity, the RN-manual dexterity relation was not statistically independent of the stronger behavioral correlation exhibited by the GP.

Magnetic susceptibility of gray matter nuclei is predominantly determined by the level of iron concentration. Our finding that susceptibility of GP is negatively correlated with motor function in healthy adults is consistent with prior findings that iron accumulates in specific gray matter regions and that iron overload is associated with functional decline during aging and in neurological diseases (Zecca et al., 2004). Another study also reported that a calorie-restricted diet can decrease brain iron accumulation and preserve motor performance (Kastman et al., 2010). While the biological mechanism of age-related iron accumulation is unknown, the negative correlation between susceptibility and motor function is not simply due to age, as susceptibility of GP was not significantly correlated with age in our cohort, and our analyses statistically controlled for age-related effects in all ROIs. Further, our analyses controlled statistically for ROI size in all analyses. Our findings

Table 4

Magnetic susceptibility within regions of interest as predictors of manual dexterity factor.

\begin{tabular}{lrlc}
\hline Predictor & \multicolumn{1}{l}{ B } & \multicolumn{1}{l}{ SE B } & \multicolumn{1}{l}{ B } \\
\hline Age & -0.04769 & 0.00692 & $-6.89^{* * *}$ \\
Gender & 0.04905 & 0.07599 & 0.65 \\
MMSE & 0.02928 & 0.07241 & 0.40 \\
Putamen & 0.04019 & 0.09442 & 0.43 \\
Globus pallidus & -0.18823 & 0.08869 & $-2.12^{*}$ \\
Red nuclei & -0.15945 & 0.09520 & -1.67 \\
Substantia nigra & 0.08789 & 0.08833 & 0.99 \\
Dentate nuclei & 0.02321 & 0.08224 & 0.28 \\
Caudate nuclei & 0.06669 & 0.08033 & 0.83
\end{tabular}

Note: $n=132$. All predictor variables entered simultaneously. Factor scores for manual dexterity were obtained from the first factor in the exploratory factor analysis of the 11 behavioral measures (Table 2). Magnetic susceptibility values were residualized for the effects of region size. MMSE $=$ Mini-Mental State Exam.

$* p<0.05$.

*** $p<0.001$ thus indicate that the negative correlation between susceptibility of GP and motor function is likely due to the accumulation of iron.

A limitation of this study is that magnetic susceptibility is highly correlated across the ROIs. As a result, the selection of ROIs and the participant inclusion may influence the results of the statistical analysis. In this study, the ROIs were created and refined before statistical analysis. All 132 participants were included in the analysis as all are clinically considered healthy. Although global cognitive function as represented by MMSE was controlled statistically, 14 participants had MMSE scores $<27$, and further research should investigate whether the relation between magnetic susceptibility and behavioral performance varies in relation to cognitive status. Another limitation is that the behavioral factors in Fig. 4 are specific to individual tests. All the variables comprising the first factor came from Purdue pegboard, and the second factor comprised only two variables, both of which came from WCST. Thus, the factors do not represent general cognitive abilities beyond these individual tests. Further, Purdue pegboard contributed more variables to the factor analysis than the other tests, which may have influenced its role as the primary factor. Thus, studies with more behavioral tests and more subjects may help us further investigate and confirm our findings.

Our results also showed that magnetic susceptibilities of the iron rich nuclei are not correlated with blood iron, transferrin or ferritin (Table 6). These results are reasonable given the multiple regulating factors during iron transport, uptake and storage. As such, neuroimaging methods, such as QSM, can offer more accurate means to investigate the effect of iron overload on the neurological system than the peripheral blood measurements. As iron overload has been suggested to be involved in mechanisms underlying major neurological disorders, such as Alzheimer's disease, Parkinson's disease, and Huntington's disease (Kandel and Schwartz, 1985; Zecca et al., 2004), this type of correlation

Table 5

Magnetic susceptibility within regions of interest as predictors of executive function factor.

\begin{tabular}{lclr}
\hline Predictor & Parameter estimate $(B)$ & Standard error $(S E B)$ & \multicolumn{1}{c}{$t$} \\
\hline Age & -0.00770 & 0.00784 & -0.98 \\
Gender & 0.06041 & 0.08605 & 0.70 \\
MMSE & 0.11579 & 0.08199 & 1.41 \\
Putamen & 0.02632 & 0.10691 & 0.25 \\
Globus pallidus & -0.01583 & 0.10042 & -0.16 \\
Red nuclei & -0.04220 & 0.10779 & -0.39 \\
Substantia nigra & -0.03796 & 0.10002 & -0.38 \\
Dentate nuclei & -0.13380 & 0.09312 & -1.44 \\
Caudate nuclei & 0.08486 & 0.09095 & 0.93 \\
\hline
\end{tabular}

Note: $n=132$. All predictor variables entered simultaneously. Factor scores for executive function were obtained from the second factor in the exploratory factor analysis of the 11 behavioral measures (Table 2). Magnetic susceptibility values were residualized for the effects of region size. MMSE = Mini-Mental State Exam.

No predictors were significant at $p<0.05$. 
Table 6

Correlation between magnetic susceptibility, for each region of interest, with peripheral iron biomarkers.

\begin{tabular}{lrlrllr}
\hline & PU & GP & \multicolumn{1}{l}{ RN } & \multicolumn{1}{l}{ SN } & \multicolumn{1}{l}{ DN } & \multicolumn{1}{l}{ CN } \\
\hline Blood iron & 0.112 & 0.018 & -0.041 & -0.064 & 0.147 & 0.105 \\
Transferrin & -0.190 & 0.021 & 0.019 & -0.047 & 0.107 & 0.013 \\
Ferritin & -0.102 & 0.088 & 0.069 & -0.017 & 0.121 & -0.037
\end{tabular}

Note: $n=124$. Values are Pearson $r$ correlations, partialed for the effects of age and gender. PU = Putamen; GP = Globus Pallidus; RN = Red Nuclei; SN = Substantia Nigra; $\mathrm{DN}=$ Dentate Nuclei; $\mathrm{CN}=$ Caudate. No correlations were significant at $p<0.05$.

studies would open a new avenue to investigate the roles of iron accumulation during neurodegeneration.

In conclusion, we found that the manual dexterity, as measured by the latent variables of behavioral tests, is negatively correlated with magnetic susceptibility in the GP. As GP contains the highest amount of non-heme iron in the brain, this result suggests that magnetic susceptibility of the GP is a potential marker of motor function decline. Our results also support the added value of QSM in studying the impact of iron deposition on brain function compared to simply using the levels of peripheral blood iron or iron-related transport or storage proteins.

\section{Acknowledgment}

The study is supported by the National Institutes of Health (NIH) through grant R01 MH096979 to C. L. C. L. and A.W. S. are also, in part, supported by NIH grants EB009483, NS075017, and R01 AG039684 (DJM). Christian Langkammer is supported by the Austrian Science Fund (FWF, project number P23576). Wei Li appreciates discussions with Jack Lancaster, PhD, from the University of Texas Health Science Center at San Antonio.

\section{References}

Aquino, D., Bizzi, A., Grisoli, M., Garavaglia, B., Bruzzone, M.G., Nardocci, N., Savoiardo, M., Chiapparini, L., 2009. Age-related iron deposition in the basal ganglia: quantitative analysis in healthy subjects1. Radiology 252, 165-172.

Bilgic, B., Pfefferbaum, A., Rohlfing, T., Sullivan, E.V., Adalsteinsson, E., 2012. MRI estimates of brain iron concentration in normal aging using quantitative susceptibility mapping. Neurolmage 59, 2625-2635.

Buja, A., Eyuboglu, N., 1992. Remarks on parallel analysis. Multivar. Behav. Res. 27, 509-540.

Burdo, J.R., Connor, J.R., 2003. Brain iron uptake and homeostatic mechanisms: an overview. Biometals 16, 63-75.

de Rochefort, L., Liu, T., Kressler, B., Liu, J., Spincemaille, P., Lebon, V., Wu, J.L., Wang, Y. 2010. Quantitative susceptibility map reconstruction from MR phase data using Bayesian regularization: validation and application to brain imaging. Magn. Reson. Med. 63, 194-206.

Deistung, A., Schäfer, A., Schweser, F., Biedermann, U., Turner, R., Reichenbach, J.R., 2013. Toward in vivo histology: a comparison of quantitative susceptibility mapping (QSM) with magnitude-, phase-, and R2*-imaging at ultra-high magnetic field strength. Neurolmage 65, 299-314.

Emerit, J., Beaumont, C., Trivin, F., 2001. Iron metabolism, free radicals, and oxidative injury. Biomed. Pharmacother. 55, 333-339.

Folstein, M.F., Folstein, S.E., McHugh, P.R., 1975. "Mini-mental state": a practical method for grading the cognitive state of patients for the clinician. J. Psychiatr. Res. 12, 189-198.

Gelman, N., Gorell, J.M., Barker, P.B., Savage, R.M., Spickler, E.M., Windham, J.P., Knight, R.A., 1999. MR imaging of human brain at 3.0T: preliminary report on transverse relaxation rates and relation to estimated iron content. Radiology 210, 759-767.

Gruber, P., Gould, D., 2010. The red nucleus: past, present, and future. Neuroanatomy 9, 1-3.

Haacke, E.M., Chengb, N.Y.C., House, M.J., Liu, Q., Neelavalli, J., Ogg, R.J., Khan, A., Ayaz, M., Kirsch, W., Obenaus, A., 2005. Imaging iron stores in the brain using magnetic resonance imaging. Magn. Reson. Imaging 23, 1-25.

Hallgren, B., Sourander, P., 1958. The effect of age on the non-haemin iron in the human brain. J. Neurochem. 3, 41-51.

Heaton, R.K., Chelune, G.J., Talley, J.L., Kay, G.G., Curtis, G., 1993. Wisconsin Card Sorting Test (WCST) Manual Revised and Expanded. Psychological Assessment Resources, Odessa, FL.

Jodzio, K., Biechowska, D., 2010. Wisconsin card sorting test as a measure of executive function impairments in stroke patients. Appl. Neuropsychol. 17, 267-277.
Kandel, E.R., Schwartz, J.H. (Eds.), 1985. Principles of Neurual Science. Elsevier Science, New York.

Kastman, E.K., Willette, A.A., Coe, C.L., Bendlin, B.B., Kosmatka, K.J., McLaren, D.G., Xu, G., Canu, E., Field, A.S., Alexander, A.L., 2010. A calorie-restricted diet decreases brain iron accumulation and preserves motor performance in old rhesus monkeys. J. Neurosci. 30, 7940-7947.

Langkammer, C., Schweser, F., Krebs, N., Deistung, A., Goessler, W., Scheurer, E., Sommer K., Reishofer, G., Yen, K., Fazekas, F., Ropele, S., Reichenbach, J.R., 2012. Quantitative susceptibility mapping (QSM) as a means to measure brain iron? A post mortem validation study. Neurolmage 62, 1593-1599.

Lewis, M.M., Du, G., Kidacki, M., Patel, N., Shaffer, M.L., Mailman, R.B., Huang, X., 2013. Higher iron in the red nucleus marks Parkinson's dyskinesia. Neurobiol. Aging 34, 1497-1503.

Lezak, M.D., 1995. Neuropsychological Assessment. Oxford University Press, Oxford.

Li, W., Wu, B., Liu, C., 2011. Quantitative susceptibility mapping of human brain reflects spatial variation in tissue composition. Neurolmage 55, 1645-1656.

Li, W., Avram, A.V., Wu, B., Xiao, X., Liu, C., 2014a. Integrated Laplacian-based phase unwrapping and background phase removal for quantitative susceptibility mapping. NMR Biomed. 27, 219-227.

Li, W., Wu, B., Batrachenko, A., Bancroft-Wu, V., Morey, R.A., Shashi, V., Langkammer, C. Bellis, M.D.D., Ropele, S., Song, A.W., Liu, C., 2014b. Differential developmental trajectories of magnetic susceptibility in human brain gray and white matter over the lifespan. Hum. Brain Mapp. 35, 2698-2713.

Liu, T., Spincemaille, P., de Rochefort, L., Kressler, B., Wang, Y., 2009. Calculation of susceptibility through multiple orientation sampling (COSMOS): a method for conditioning the inverse problem from measured magnetic field map to susceptibility source image in MRI. Magn. Reson. Med. 61, 196-204.

Lotfipour, A.K., Wharton, S., Schwarz, S.T., Gontu, V., Schäfer, A., Peters, A.M., Bowtell, R.W. Auer, D.P., Gowland, P.A., Bajaj, N.P.S., 2012. High resolution magnetic susceptibility mapping of the substantia nigra in Parkinson's disease. J. Magn. Reson. Imaging 35, 48-55.

Moos, T., Morgan, E.H., 2004. The metabolism of neuronal iron and its pathogenic role in neurological disease: review. Ann. N. Y. Acad. Sci. 1012, 14-26.

Nioche, C., Cabanis, E.A., Habas, C., 2009. Functional connectivity of the human red nucleus in the brain resting state at 3T. Am. J. Neuroradiol. 30, 396-403.

War Department Adjutant General's Office, 1944. Army individual test battery. Manual of Directions and Scoring. United States War Department, Washington, DC.

Rauscher, A., Sedlacik, J., Barth, M., Mentzel, H.J., Reichenbach, J.R., 2005. Magnetic susceptibility-weighted MR phase imaging of the human brain. Am. J. Neuroradiol. 26, 736-742.

Reitan, R.M., Wolfson, D., 1985. The Halstead-Reitan Neuropsychological Test Battery: Therapy and Clinical Interpretation. Neuropsychological Press, Tucson, AZ.

Schmidt, R., Fazekas, F., Kapeller, P., Schmidt, H., Hartung, H.-P., 1999. MRI white matter hyperintensities three-year follow-up of the Austrian Stroke Prevention Study. Neurology 53, 132-132.

Schmidt, R., Enzinger, C., Ropele, S., Schmidt, H., Fazekas, F., 2003. Progression of cerebral white matter lesions: 6-year results of the Austrian Stroke Prevention Study. Lancet 361, 2046-2048.

Schmidt, R., Ropele, S., Enzinger, C., Petrovic, K., Smith, S., Schmidt, H., Matthews, P.M. Fazekas, F., 2005. White matter lesion progression, brain atrophy, and cognitive decline: the Austrian Stroke Prevention Study. Ann. Neurol. 58, 610-616.

Schweser, F., Deistung, A., Lehr, B.W., Reichenbach, J.R., 2011. Quantitative imaging of intrinsic magnetic tissue properties using MRI signal phase: an approach to in vivo brain iron metabolism? NeuroImage 54, 2789-2807.

Shmueli, K., de Zwart, J.A., van Gelderen, P., Li, T.Q., Dodd, S.J., Duyn, J.H., 2009. Magnetic susceptibility mapping of brain tissue in vivo using MRI phase data. Magn. Reson. Med. 62, 1510-1522.

Smith, S.M., 2002. Fast robust automated brain extraction. Hum. Brain Mapp. 17, $143-155$.

Sullivan, E.V., Adalsteinsson, E., Rohlfing, T., Pfefferbaum, A., 2009. Relevance of iron deposition in deep gray matter brain structures to cognitive and motor performance in healthy elderly men and women: exploratory findings. Brain Imaging Behav. 3, 167-175.

ten Donkelaar, H.J., 1988. Evolution of the red nucleus and rubrospinal tract. Behav. Brain Res. 28, 9-20.

Tiffin, J., Asher, E.J., 1948. The Purdue pegboard: norms and studies of reliability and validity. J. Appl. Psychol. 32, 234.

Wechsler, D., 2003. Wechsler intelligence scale for children, Administration and Scoring ManualFourth Edition. Harcourt Assessment, Inc, San Antonio, TX.

Wharton, S., Schafer, A., Bowtell, R., 2010. Susceptibility mapping in the human brain using threshold-based k-space division. Magn. Reson. Med. 63, 1292-1304.

Wu, B., Li, W., Guidon, A., Liu, C., 2012. Whole brain susceptibility mapping using compressed sensing. Magn. Reson. Med. 67, 137-147.

Yao, B., Li, T.-Q., Pv, Gelderen, Shmueli, K., de Zwart, J.A., Duyn, J.H., 2009. Susceptibility contrast in high field MRI of human brain as a function of tissue iron content. NeuroImage 44, 1259-1266.

Zecca, L., Youdim, M.B., Riederer, P., Connor, J.R., Crichton, R.R., 2004. Iron, brain ageing and neurodegenerative disorders. Nat. Rev. Neurosci. 5, 863-873. 